\title{
Perioperative duloxetine as part of a multimodal analgesia regime reduces postoperative pain in lumbar canal stenosis surgery: a randomized, triple blind, and placebo-controlled trial
}

\author{
Nishith Govil ${ }^{1}$, Kumar Parag $^{2}$, Pankaj Arora ${ }^{3}$, Hariom Khandelwal ${ }^{2}$, Ashutosh Singh $^{2}$, and Ruchi ${ }^{4}$ \\ 'Department of Anaesthesiology, All India Institute of Medical Sciences Rishikesh, Rishikesh, India \\ 2Department of Anaesthesiology, Shri Guru Ram Rai Institute of Medical and Health Sciences, Dehradun, India \\ ${ }^{3}$ Department of Neurosurgery, Shri Guru Ram Rai Institute of Medical and Health Sciences, Dehradun, India \\ ${ }^{4}$ Department of Community Medicine, Government Doon Medical College, Dehradun, India
}

Received June 4, 2019

Revised August 19, 2019

Accepted August 26, 2019

\section{Correspondence \\ Nishith Govil \\ Department of Anaesthesiology, All India Institute of Medical Sciences Rishikesh, Virbhadra Road Near Barrage, Shivaji Nagar, Sturida Colony, Rishikesh 249203, India \\ Tel: +91-0135-2752946 \\ Fax: +91-0135-0161340 \\ E-mail: nishithgovil@rediffmail.com}

Previous presentation at conferences: The 20th Society of Anaesthesiologists of Nepal Annual Conference (SANCON 2019), 14-16th Mar. 2019, Kathmandu, Nepal.
Background: Duloxetine is an antidepressant that is also useful in chronic neuropathic and central origin pain. In this study, the role of duloxetine in decreasing acute postoperative pain after lumbar canal stenosis surgery is explored.

Methods: In this single center, triple blinded, and placebo-controlled trial, 96 patients were randomized for statistical analysis. The intervention group received oral duloxetine $30 \mathrm{mg}$ once a day (OD) for 2 days before surgery, $60 \mathrm{mg} O D$ from the day of surgery to the postoperative second day and $30 \mathrm{mg} O D$ for the next 2 days (a total duration of 7 days). A placebo capsule was given in the other group for a similar time and schedule. The same standard perioperative analgesia protocols were followed in both groups.

Results: Total morphine consumption up to 24 hours was significantly decreased in the duloxetine group $(P<0.01)$. The time to the first analgesia requirement was similar in both groups but the time to the second and third dose of rescue analgesia increased significantly in the duloxetine group. The time to ambulation was decreased significantly $(P<0.01)$ in the duloxetine group as compared to the placebo group. Pain scores remained similar during most of the time interval. No significant difference was observed in the complication rate and patient satisfaction score recorded.

Conclusions: Duloxetine reduces postoperative pain after lumbar canal stenosis surgery with no increase in adverse effects.

Key Words: Acute Pain; Analgesia; Antidepressive Agents; Duloxetine Hydrochloride; Humans; Morphine; Pain Management; Pain, Postoperative; Patient Satisfaction; Walking

\section{INTRODUCTION}

Postoperative pain after lumbar canal stenosis (LCS) surgery involves a pre-existing neuropathic component as well as a nociceptive component. A majority of these pa- tients were already on opioids or nonopioid analgesics before surgery. Conventional analgesics sometimes may not be able to alleviate the post-surgical pain (PSP) completely [1]. It is known that inadequate treatment of PSP within 48 hours is a risk factor for the development of chronic PSP (a) This is an open-access article distributed under the terms of the Creative Commons Attribution Non-Commercial License (http://creativecommons.org/licenses/by-nc/4.0/), which permits unrestricted non-commercial use, distribution, and reproduction in any medium, provided the original work is properly cited.

(c) The Korean Pain Society, 2020
Author contributions: Nishith Govil: Investigation; Kumar Parag: Writing/ manuscript preparation; Pankaj Arora: Investigation; Hariom Khandelwal: Writing/manuscript preparation; Ashutosh Singh: Writing/manuscript preparation; Ruchi: Formal analysis. 
and newer drugs are an emerging alternative [2].

Duloxetine is a serotonin and norepinephrine (NE) reuptake inhibitor for modulation of pain in the central nervous system. A number of well-conducted randomized studies have examined the efficacy and safety of duloxetine in major depressive disorders, painful diabetic neuropathy, and the chronic pain of fibromyalgia [3]. Evidence is growing in the favour of duloxetine as compared to a placebo or in combinations with other adjuvants for treating PSP with fewer side effects. To evaluate the role of duloxetine in the acute management of pain after LCS surgery we decided to conduct and report this trial.

\section{MATERIALS AND METHODS}

\section{Study design}

This single center randomized, triple blinded, and placebo-controlled trial was approved by the Ethical Committee of the Shri Guru Ram Rai Institute of Medical and Health Sciences where the study was conducted (vide letter number SGRR/IEC/03/17 dated 05/01/2017). The study was conducted over a period of 8 months (January 2017 to August 2017) as per the Helsinki declaration on human experimentation. This institute is a 1,200-bed teaching hospital with specialization in spine surgeries and a wellestablished pain and palliative care department.

Written, informed, and explained consent taken from patients before participation in the study. The study design and reporting conforms to the consolidated standards of reporting trials (CONSORT) standards. All the eligible patients enrolled in the study after assessing the inclusion and exclusion criteria.

\section{Study participants}

Patients of age between 18 and 60 years of either sex, American Society of Anesthesiologists (ASA) grade I-II undergoing decompression and/or instrumentation for lumbar radiculopathy due to LCS were included in the study. Exclusion criteria were known allergies to the test drug, an abnormal liver and renal function test, uncontrolled narrow angle glaucoma, being a chronic opioid abuser (>3 mo), being on chronic gabapentin and or pregabalin (>3 mo), and being on monoamine oxidase inhibitor, tricyclic antidepressant, or fluvoxamine. Pregnant females, patients with psychiatric disorders or seizure disorders, and known smokers were excluded from the study.

\section{Study intervention}

Patients were randomly allocated into two group using computer generated tables of random numbers concealed in serially numbered opaque sealed envelopes which were opened by the councillor (blinded to the group allocation) on the day when the patients got registered for the surgery. The patient, treating physician, and evaluator were unaware of the group assignments of the patients.

Patients were enrolled two days before the day of surgery and divided into two parallel arms. Group A received oral duloxetine (Duzela $30 \mathrm{mg}$ delayed release capsule; Sun Pharma Company, Mumbai, India) $30 \mathrm{mg}$ once a day (OD) for 2 days before surgery. The dose was incremented to $60 \mathrm{mg}$ OD from the day of surgery (postoperative day [POD] 0) to the second postoperative day (POD 2), again tapered to $30 \mathrm{mg}$ OD for the next 2 days, i.e. until POD 5 (total duration 7 days), and then stopped. Duloxetine was given at a fixed time in the morning every day to increase compliance. A similar looking placebo capsule was prepared and given to the patients in Group B for the same duration and schedule. The dose of duloxetine in this and previous studies was selected on the basis that $120 \mathrm{mg}$ daily dosing causes more side effects than a $60 \mathrm{mg}$ dose, with comparable analgesic effects [3].

All previous analgesic drugs were stopped 2 days before the surgery and patients were asked to take tablet acetaminophen $325 \mathrm{mg}$ for any breakthrough pain. The study protocol was explained to patients, including the 11-point numerical rating scale (NRS) and the common side effects of duloxetine, such as nausea, gastric irritation, insomnia, drowsiness, and dry mouth.

\section{Perioperative anesthesia protocol}

Patients in both groups were operated on by the same neurosurgeons that performed all the surgeries in the study. The same standard general anesthesia protocols were followed in all patients. Induction was done with injection propofol and injection fentanyl $(2 \mu \mathrm{g} / \mathrm{kg})$, and intubation was facilitated with injection rocuronium. An oxygennitrous oxide mixture with isoflurane concentration was adjusted to keep minimum alveolar concentration 1.2 for maintenance of anesthesia. Fentanyl $1 \mu \mathrm{g} / \mathrm{kg}$ was given for intraoperative pain. After an adequate reversal of muscle relaxation with neostigmine and glycopyrrolate, the patient was smoothly extubated. Patients stayed in the post anesthesia care unit (PACU) for 24 hours where intravenous morphine $0.05 \mathrm{mg} / \mathrm{kg}$ was given if heart rate and mean arterial the pressure rose above $30 \%$ of baseline or NRS $\geq 4$. Afterwards, a 24-hour oral acetaminophen $325 \mathrm{mg}$ and tramadol $37.5 \mathrm{mg}$ drug combination was given 
twice a day for pain until POD 7. Following no complication, patients were discharged from the hospital with follow-up advised after 1 week.

\section{Outcomes measured}

Primary outcomes measured were total morphine (rescue analgesia) consumed in 24 hours and time to rescue analgesia (morphine) requirement up to 24 hours postoperatively. Secondary outcomes measured were time to ambulation and pain scores (using the NRS, with 0 being no pain and 10 as the worst imaginable pain).

In the PACU, patients were monitored for pain using the NRS scale at 0 (immediately postoperative), 1, 2, 4, 8, 12, 24, 36 , and 48 hours postoperatively, as well as at ambulation. A nurse blinded to the study recorded NRS scores and total morphine consumed up to 24 hours. Time to first, second, and third analgesia requirements and time to ambulation were also recorded. At the follow-up (on POD 7), a patient satisfaction score on a 4 point Likert scale (excellent, good, fair, poor) and complications like sedation or agitation, dry mouth, episodes of nausea and vomiting or any other adverse events were recorded.

\section{Statistical analysis}

Sample size was calculated, based on a previous study where patient received a similar postoperative analgesic regime in same cohort of spine surgeries [4]. In that study mean total morphine consumption was $42.80 \pm 10.90$ in placebo group and $16.30 \pm 8.90$ in intervention group at 24 hours. Assuming a common within-group standard devia- tion (SD) of 2 points, a power of study of $80 \%$, and a 2 -sided alpha level of 0.05 to detect a $33 \%$ difference in morphine consumption between the two groups, we needed to enroll 46 patients in each group. To account for attrition, we decided to enroll 52 patients in each group.

Statistical tests were conducted on SPSS Statistics ver. 23 (IBM Corp., Armonk, NY). Normalcy of data was checked with a Shapiro-Wilk test. Normally distributed data was presented as mean \pm SD while non-normally distributed data was presented as Median (interquartile range). Numbers were taken as percentages and proportions. An unpaired $t$-test was used to compare the means, a MannWhittney test was used to compare the medians, and a chi-square test was used to compare percentages. $P<0.05$ considered statistically significant. Graphs were formed using Microsoft Excel spreadsheets (Microsoft, Redmond, WA), while the survival curve was drawn using a log rank test for comparing two groups on SPSS software.

\section{RESULTS}

We assessed 104 patients for eligibility to be included in the study. Eight patients were excluded from enrollment and 96 patients were randomized into 2 groups; Group A (the duloxetine group) and Group B (the placebo group). In the final analysis, 92 patients were included for statistical analysis and inference (Fig. 1).

The demographic variables of the patients as well as the intraoperative parameters were similar in both groups (Table 1). Total morphine consumption (mean \pm SD) up to 24 hours was significantly decreased in the duloxetine
CONSORT 2010 flow diagram

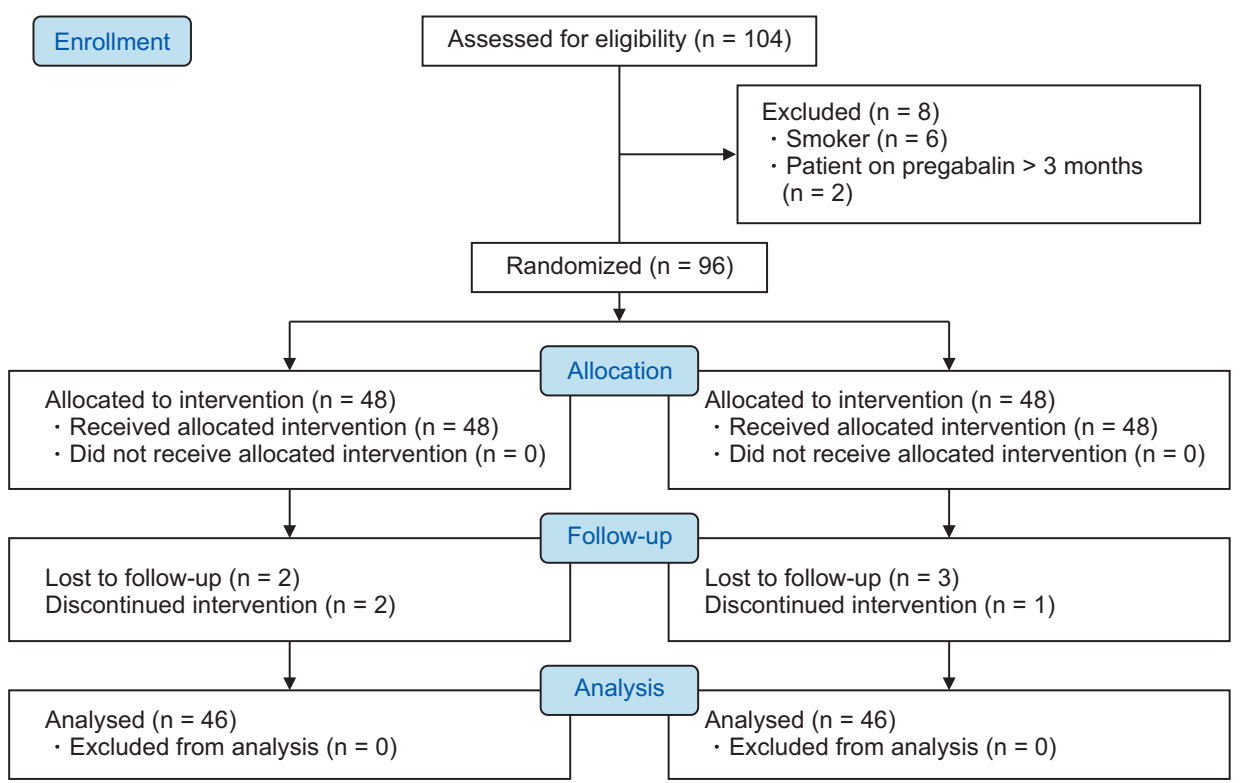

Korean J Pain 2020;33(1):40-47
Fig. 1. CONSORT flow diagram.

https://doi.org/10.3344/kjp.2020.33.1.40 
group (10.43 $\pm 1.51 \mathrm{mg}$ vs. $12.55 \pm 1.74 \mathrm{mg} ; 95 \%$ confidence interval [CI], -2.80 to $-1.42 ; P<0.01$ ). Time to first analgesia requirement (mean $\pm \mathrm{SD}$ ) was similar in both groups $(1.49 \pm 0.62$ hr vs. $1.32 \pm 0.44 \mathrm{hr} ; 95 \% \mathrm{CI},-0.59$ to $0.40 ; P$ $=0.14)$. Time to ambulation was decreased significantly (95\% CI, -21.82 to $-18.83 ; P<0.01$ ) in the duloxetine group (mean $\pm \mathrm{SD}, 25.09 \pm 4.12 \mathrm{hr}$ ) as compared to the placebo group (mean \pm SD, $45.45 \pm 2.60 \mathrm{hr}$, Table 2).

The test of equality of survival distributions for the different levels of the duloxetine group (Log Rank MantelCox test $\chi^{2}=10.56$, degree of freedom $1, P<0.01$ ) show significantly increased time to the second and third dose of rescue analgesia requirement (Figs. 2, 3). Postoperative NRS scores remained similar during most of the time interval (Table 3). No significant difference was observed in the complication rate (recorded at the 24th postoperative hour and on the 7th POD) and patient satisfaction score (Table 4).

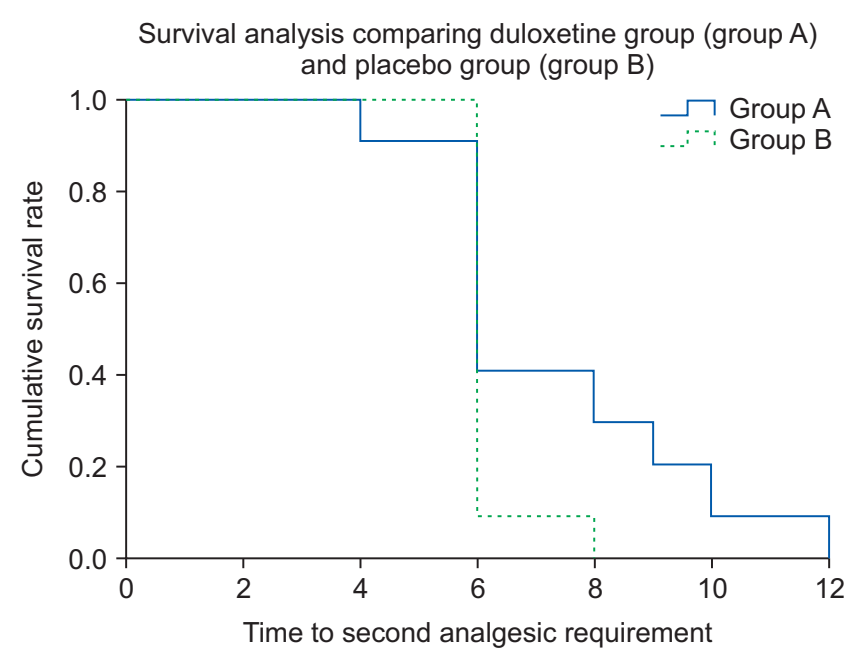

Fig. 2. Test of equality of survival distributions for the different levels of group for time to second analgesic requirement show significant difference (degree of freedom 1) with Log Rank (Mantel-Cox).

Table 1. Baseline Demographic Variables

\begin{tabular}{lcccc}
\hline \multicolumn{1}{c}{ Parameter } & Group A & Group B & $\begin{array}{c}\text { Mean difference } \\
\text { (95\% Cl) }\end{array}$ & $\begin{array}{c}P \text { value } \\
\text { Standardized } \\
\text { difference }\end{array}$ \\
\hline Age (yr) & $41.40 \pm 14.60$ & $43.00 \pm 15.10$ & $-1.63(-7.93$ to 4.65$)$ & 0.60 \\
Sex (male/female) & $23(50.00) / 23(50.00)$ & $22(47.83) / 24(52.17)$ & -0.51 & 0.67 \\
ASA grade I & $20(43.48)$ & $18(39.13)$ & 0.67 \\
ASA grade II & $26(56.52)$ & $28(60.87)$ & & 0.68 \\
BMl (kg/m ${ }^{2}$ ) & $25.90 \pm 2.30$ & $26.60 \pm 2.20$ & $-0.71(-1.67$ to 0.24$)$ & 0.14 \\
Concurrent analgesic use & & & -1.48 \\
$\quad$ WHO ladder I & $21(45.65)$ & $19(41.30)$ & & 0.67 \\
$\quad$ WHO ladder II & $25(54.35)$ & $27(58.70)$ & & 0.68 \\
Underlying disease duration (day) & $31.00 \pm 4.03$ & $30.60 \pm 6.19$ & $-0.40(-2.56$ to 1.76$)$ & 0.71 \\
Duration of surgery (hr) & $1.89 \pm 0.33$ & $1.80 \pm 0.37$ & $0.94(-0.56$ to 0.24$)$ & 0.21 \\
Intraoperative fentanyl use & $217.50 \pm 42.57$ & $215.00 \pm 45.95$ & $-2.50(-20.85$ to 15.85) & 0.78 \\
\hline
\end{tabular}

Values are presented as mean \pm standard deviation or number (\%).

Group A: the duloxetine group, Group B: the placebo group, Cl: confidence interval, ASA: American Society of Anesthesiologists, BMI: body mass index, WHO: World Health Organization.

${ }^{a}$ Standardized difference $=$ difference in means or proportions divided by standard error; imbalance defined as absolute value greater than 0.20 (small effect size).

Table 2. Comparison of time to Rescue Analgesia Requirement (Morphine) in First 24 Hours Postoperatively

\begin{tabular}{|c|c|c|c|c|c|}
\hline Parameter & Group A & Group B & $\begin{array}{c}\text { Mean difference } \\
\text { (95\% Cl of difference) }\end{array}$ & $\begin{array}{c}\text { Log Rank } \\
(\text { Mantel-Cox })^{a}\end{array}$ & $P$ value \\
\hline Total morphine consumption in 24-hr (mg) & $10.43 \pm 1.51$ & $12.55 \pm 1.74$ & $-2.12(-2.80$ to -1.42$)$ & & $<0.01$ \\
\hline Time to I analgesic requirement (hr) & $1.49 \pm 0.62$ & $1.32 \pm 0.44$ & $0.17(-0.59$ to 0.40$)$ & 1.40; 1 & 0.14 \\
\hline Time to II analgesic requirement (hr) & $7.32 \pm 2.26$ & $6.18 \pm 0.58$ & $1.13(0.43$ to 1.83$)$ & $10.55 ; 1$ & $<0.01$ \\
\hline Time to III analgesic requirement (hr) & $19.77 \pm 3.74$ & $12.18 \pm 0.58$ & 7.59 (6.45 to 8.72$)$ & $88.79 ; 1$ & $<0.01$ \\
\hline Time to ambulation (hr) & $25.09 \pm 4.12$ & $45.45 \pm 2.60$ & $-20.36(-21.82$ to -18.83$)$ & 39.66; 1 & $<0.01$ \\
\hline
\end{tabular}

Values are presented as mean \pm standard deviation. $P<0.05$ is considered statistically significant.

Group A: the duloxetine group, Group B: the placebo group, Cl: confidence interval.

${ }^{a} \chi^{2}$; degree of freedom. 


\section{DISCUSSION}

Duloxetine administration in LCS surgery lead to decreased morphine consumption up to 24 hours postoperatively and increased time to rescue analgesia (primary outcome). Duloxetine also decreased time to ambulation after LCS surgery without any increase in adverse effects. Pain scores remained similar in both groups at most of the time intervals. Results from our study support our hypothesis that duloxetine can be used for postoperative pain relief.

\section{Duloxetine as study drug}

Duloxetine is an antidepressant that is also useful in chronic neuropathic and central origin pain. Three modes and sites have been postulated for duloxetine to exert its analgesic effects. It acts at the spinal cord level by

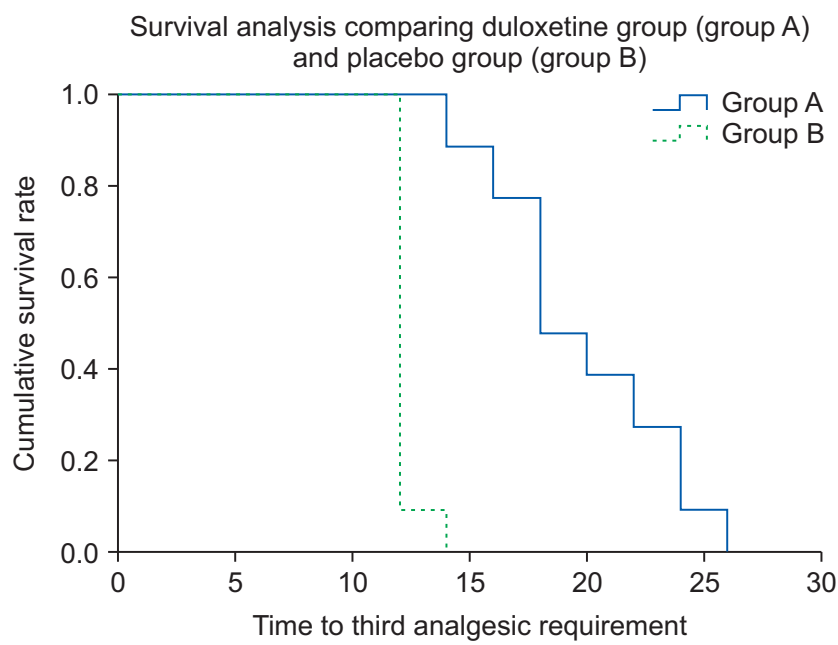

Fig. 3. Test of equality of survival distributions for the different levels of group for time to third analgesic requirement show significant difference (degree of freedom 1) with Log Rank (Mantel-Cox). increasing the level of the neurotransmitters NE, dopamine, and serotonin in the dorsal horn of the spinal cord. These monoamines activate spinal $5-\mathrm{HT}_{2} \mathrm{~A}$ and aplha2noradrenergic receptors that potentiate inhibitory descending pain pathways in the spinal cord. Another central mechanism is the activation of the prefrontal cortex, which causes cognitive modulation of pain. Duloxetine's peripheral action as a local anaesthetic is due to blockage of Neuronal Navl.7 $\mathrm{Na}^{+}$channel [5].

To confirm the site of the action of duloxetine, Sun et al. [6] gave duloxetine intrathecally and intraperitoneally in a

Table 4. Adverse Events and Patient Satisfaction Score on a 4-Point Likert Scale (Excellent, Good, Fair, and Poor)

\begin{tabular}{llll}
\hline \multicolumn{1}{c}{ Parameter } & Group A & Group B & $P$ value \\
\hline Complication on postoperative 24th hr & & & \\
Presence of nausea & $3(6.52)$ & $4(8.70)$ & 0.70 \\
Vomiting episodes 0-2 times & $5(10.87)$ & $6(13.04)$ & 0.75 \\
Vomiting episodes 2-4 times & $2(4.35)$ & $4(8.70)$ & 0.40 \\
Vomiting episodes > 4 times & 0 & $1(2.17)$ & 0.31 \\
Bradycardia & $1(2.17)$ & $1(2.17)$ & 1.00 \\
Hypotension & 0 & 0 & - \\
Somnolence & $3(6.52)$ & $4(8.70)$ & 0.70 \\
Complication up to postoperative day 7 & & & \\
Nausea/vomiting episodes & $10(21.74)$ & $6(13.04)$ & 0.03 \\
Gastritis & $2(4.35)$ & $2(4.35)$ & 0.47 \\
Dry mouth & $6(13.04)$ & $2(4.35)$ & 0.001 \\
Somnolence & $1(2.17)$ & $1(2.17)$ & 0.55 \\
Likert scale class & & & \\
Excellent & $18(39.13)$ & $16(34.78)$ & 0.23 \\
Good & $12(26.09)$ & $14(30.43)$ & 0.34 \\
Fair & $10(21.74)$ & $12(26.09)$ & 0.34 \\
Poor & $6(13.04)$ & $4(8.70)$ & 0.36 \\
\hline
\end{tabular}

Values are presented as number (\%). $P<0.05$ is considered statistically significant.

Group A: the duloxetine group, Group B: the placebo group.

Table 3. Comparison of Postoperative Pain Scores

\begin{tabular}{|c|c|c|c|c|}
\hline Parameter & Group A & Group B & $\begin{array}{c}\text { Mean difference } \\
\text { (95\% Cl of difference) }\end{array}$ & $P$ value \\
\hline Preoperative NRS & $4.8 \pm 0.8 ; 5(4-5)$ & $4.6 \pm 0.9 ; 5(4-5)$ & $0.18(-0.16$ to 0.53$)$ & 0.37 \\
\hline Postoperative $0 \mathrm{hr}$ & $1.8 \pm 0.6 ; 2(1-2)$ & $2.3 \pm 0.6 ; 2(2-3)$ & $-0.45(-0.72$ to -0.19$)$ & 0.40 \\
\hline Postoperative 1st hr & $3.6 \pm 0.9 ; 3.5(3-4)$ & $3.7 \pm 0.9 ; 4(3-4)$ & $-0.09(-0.47$ to 0.29$)$ & 0.55 \\
\hline Postoperative 2nd hr & $2.6 \pm 0.9 ; 2(2-4)$ & $2.5 \pm 1.5 ; 2(1-4)$ & $0.13(-0.38$ to 0.65$)$ & 0.14 \\
\hline Postoperative 4th hr & $2.4 \pm 0.7 ; 2(2-3)$ & $2.5 \pm 0.5 ; 2(2-3)$ & $-0.04(-0.29$ to 0.20$)$ & 0.37 \\
\hline Postoperative 6th hr & $3.5 \pm 1.1 ; 3.5(3-4)$ & $4.4 \pm 0.8 ; 4(4-5)$ & $-0.84(-1.24$ to -0.43$)$ & $<0.01$ \\
\hline Postoperative 12th hr & $2.6 \pm 0.7 ; 2.5(2-3)$ & $4.5 \pm 0.8 ; 4(4-5)$ & $-1.86(-2.17$ to -1.55$)$ & $<0.01$ \\
\hline Postoperative 24th hr & $3.1 \pm 1.0 ; 3(2-4)$ & $3.1 \pm 1.8 ; 3(2-4)$ & $-0.04(-0.64$ to 0.55$)$ & 0.56 \\
\hline Postoperative 36th hr & $3.5 \pm 0.7 ; 4(3-4)$ & $3.8 \pm 0.8 ; 4(3-4)$ & $-0.34(-0.67$ to -0.01$)$ & 0.03 \\
\hline Postoperative 48th $\mathrm{hr}$ & $2.1 \pm 0.7 ; 2(2-2)$ & $4.0 \pm 0.7 ; 4(3-4)$ & $-1.90(-2.21$ to -1.60$)$ & $<0.01$ \\
\hline NRS on ambulation & $2.7 \pm 0.6 ; 3(2-3)$ & $2.8 \pm 0.7 ; 3(2-3)$ & $-0.16(-0.45$ to 0.13$)$ & 0.31 \\
\hline
\end{tabular}

Values are presented as the mean \pm standard deviation; median (interquartile range). $P<0.05$ is considered statistically significant. Group A: the duloxetine group, Group B: the placebo group, Cl: confidence interval, NRS: numerical rating scale. 
rat model of postoperative pain. They found, using in vivo microdialysis, raised levels of serotonin (5-HT) and NE in the dorsal horn of the spinal cord. They also use an antagonist for $5-\mathrm{HT}_{2} \mathrm{~A}$ and for the alpha $\mathrm{a}_{2}$-NE receptor to partially attenuate the antihyperalgesic effects of duloxetine.

\section{LCS surgery as study model}

To study the efficacy of duloxetine, we selected patients undergoing surgery for LCS. Post-surgery nociceptive pain adds to the pre-existing neuropathic pain. Low back pain has components of both nociceptive (detected by the visual analogue scale [VAS]) and neuropathic pain (detected by the painDETECT score). Schukro et al. [7] studied the beneficial effects of duloxetine in a placebo-controlled crossover trial of 41 patients with low back pain. Duloxetine, titrated to a maximum of $120 \mathrm{mg} / \mathrm{day}$, was given for 4 weeks, followed by a washout period of 2 weeks, and a placebo for 4 weeks. The mean VAS score for the 4 weeks in the duloxetine period $(4.1 \pm 2.9)$ was significantly lower as compared to the placebo period $(6.0 \pm 2.7)$. This corresponds to an average $32 \%$ reduction in pain. Similarly, the painDETECT scores were lowered by a clinically significant number of points during the duloxetine phase of trial (17.7 \pm 5.7 vs. $21.3 \pm 3.6$ ). Thus, duloxetine seems to benefit patients in both nociceptive and neuropathic pain.

\section{Previous trials in acute pain management}

Trials exploring the potential benefits of duloxetine in relieving acute PSP exist in different surgical cohorts of patients. However, concerns have been raised against the outcomes measured, the benefits in terms of patient's functional recovery, and clinical significance of the statistically significant results in recovery scores [8]. Optimum dosing and the duration of the administration of duloxetine also affect the outcome analysed [9].

Ho et al. [10] gave two doses of duloxetine $60 \mathrm{mg}$ on successive days after surgery (knee arthroplasty). Duloxetine has been shown to be better than a placebo in terms of decreasing morphine consumption (mean $\pm \mathrm{SD}$ ) in the first 24 hours $(12.9 \pm 10.4 \mathrm{mg}$ vs. $19.8 \pm 13.7 \mathrm{mg})$ and 48 hours (19.5 $\pm 14.5 \mathrm{mg}$ vs. $30.3 \pm 18.1 \mathrm{mg})$ postoperatively. Pain scores at rest and during movement remained similar in both groups. The authors concluded that duloxetine is an effective adjuvant in preventing postoperative pain. However, they included patients undergoing knee arthroplasty under both regional and general anaesthesia, which would have affected outcomes measured the in first 24 hours. YaDeau et al. [11] studied the role of duloxetine in subacute pain (pain occurring at $2 \mathrm{wk}$ ) after knee arthroplasty. They gave $60 \mathrm{mg}$ duloxetine starting from day of surgery for 15 days and measured self-reported pain scores at the 2nd week. Secondary acute pain outcomes measured were pain scores and opioid consumption at POD 1 and POD 3. According to the authors, duloxetine did not reduce pain scores during rest and movement at POD 1, POD 3, or POD 14. However, the authors found that postoperatively opioid consumption (mean oral morphine equivalents) was significantly decreased (differences in means $8.7 \mathrm{mg} ; P=0.002$ and $95 \% \mathrm{CI}, 3.3$ to 14.1 ) up to 3 months.

\section{Primary outcome is not pain score}

Both the above studies show that pain scores remain statistically similar while opioid consumption decreases in first 24 or 48 hours after surgery. Using a primary outcome like VAS or NRS at different point of times to assess the efficacy of a drug or intervention is less useful than averaging the VAS score over a period. Examining pain scores at different times is like cross sectional analysis, which is influenced by many factors (movement, intervention like dressing, or the last dose of rescue analgesia). There are other outcomes that can be measured to study the efficacy of an intervention on acute pain. Total rescue analgesia consumed in a stipulated time, time to the first or second administration of rescue analgesia, and time to ambulation or recovery are more pragmatic and functional outcomes [12].

\section{Compliance to duloxetine intake}

In our trial, duloxetine was given for a period of 7 days, different from most other studies where duloxetine was given for 2 days. Compliance with treatment was $96 \%$ in both groups (48 out of 50) as reported by the patients and $100 \%$ till POD 5. Compliance with duloxetine intake is low as compared to a placebo when given over a long time. When duloxetine was given over a period of 2 weeks by YaDeau et al. [11], compliance in taking duloxetine at the 2nd week was $75 \%$ as compared to $85 \%$ with the placebo though the difference was not statistically significant. Similarly, Smith et al. [13] gave duloxetine $30 \mathrm{mg}$ OD for one week followed by incremented $60 \mathrm{mg}$ OD for a further 4 weeks in patients with chemotherapy induced neuropathy. They reported a drop out rate of $11 \%$ as compared to $1 \%$ in a placebo group despite similar adverse events rates in both group.

\section{Is analgesic effect of duloxetine due to its antidepressant effect?}

Castro-Alves et al. [14] gave duloxetine $60 \mathrm{mg}$ on the day of surgery and 24 hours after surgery to study the role of duloxetine in perioperative recovery after abdominal hyster- 
ectomy. The primary outcome measured was global quality of recovery-40 score (Global QoR-40) at 24-hour, which evaluated 5 aspects of patient recovery (physical comfort, physical independence, pain, emotional status, and support) ranging from 40 to 200 . The authors also compared pain scores and opioid consumption with respect to a placebo at 24- and 48-hour.

They found a statistically significant improvement of median Global QoR-40 by 9 points (95\% CI, 4-20 points) at 24-hour but not at 48-hour. Secondary outcomes in the study, pain scores and opioid consumption, were decreased significantly in the duloxetine group. The Global QoR-40 score was highly correlated to the pain subcomponent (Spearman $\rho=0.77, P<0.001$ ). The emotional status subcomponent of the Global QoR-40 at 24-hour shows a decrease of median 2 points $(P=0.0002)$ and no decrease at 48-hour $(P=0.49)$ after duloxetine intake. However, no correlation between emotional state and Global QoR-40 score was calculated in the study. Therefore, these improvements in global recovery or patient satisfaction may not be due to better analgesia control, but rather an outcome of a better physical and emotional state of comfort.

It is reasonable that if an analgesic could also provide emotional stability, it would be a better adjunct for postoperative recovery. Duloxetine also acts as an antidepressant and brings superior emotional well-being. With the help of very robust results, Minami et al. [15] has shown in a cauda equine compression neuropathic pain model in rats that the analgesic effects of duloxetine in conditions of pain are independent of its antidepressant effect and are executed at the spinal cord level.

\section{Comparison with other adjuvants}

Drugs like dexamethasone, anticonvulsants, antidepressants, nonsteroidal anti-inflammatory drugs, and centrally acting alpha 2 agonist have been tried for postoperative analgesia as sole agents or as adjuvants. Duloxetine has been also compared with some of these agents regarding its efficacy and safety profile.

Altiparmak et al. [16] compared pregabalin and duloxetine in their role as an adjuvant in a multimodal analgesia regime and postoperative effects on cognitive function after spinal surgery. Pre- and postoperative cognitive function was assessed based on the Montreal cognitive assessment (MoCA) tool. In their study, Duloxetine and pregabalin's analgesic efficacy were similar and significantly greater than a placebo. However, they found a significant reduction in mean MoCA score postoperatively (compared to the preoperative score) in pregabalin, duloxetine, and the placebo group. The highest fall in MoCA score was seen with pregabalin and the least with the control group, thus outweighing pregabalin's analgesic benefits.

Another disadvantage of pregabalin as an analgesic adjuvant as compared to duloxetine is due to the fact that the two drugs have different effects on the increased levels of monoamines in the spinal cord. Kiso et al. [17] proved, in a rat model of reserpine-induced fibromyalgia, that pregabalin increase only levels of NE in the spinal cord, while duloxetine increases both serotonin and NE. An optimal balance between NE and serotonin is important for proper analgesia and probable synergistic action with opioids.

\section{Future opportunities}

Serotonin has a differential effect on pain modulation at different concentrations by increasing or decreasing pain sensation in comparison to NE which is mostly inhibitory to pain. Duloxetine's potency to inhibit serotonin uptake is three time that of NE uptake. Shen et al. [18] showed, in a rat formalin model of nociception, that an excess of serotonin acts through $5-\mathrm{HT}_{3}$ receptors to reduce the analgesic synergy of duloxetine with morphine. It has been postulated that if duloxetine is given with ondansetron (an antagonist of the $5-\mathrm{HT}_{3}$ receptor), the analgesic synergism of duloxetine with morphine can be further increased.

\section{Limitations}

There are some limitations in our study. We did not record the NRS scores over a recovery period of one week which would have been more informative than 24 to 48 hours of the postoperative period only. Similarly, we did not assess emotional aspect or cognitive function of the patients to examine their role in pain modulation. This was a single center trial in a specific cohort of surgical patient. Further trials comparing duloxetine with other opioid-sparing analgesics or analgesic adjuvants will be required to fully understand the usefulness of duloxetine in acute pain.

Minimal use of opioids postoperatively is an important factor in enhanced recovery that is measured in terms of time to ambulation and functional recovery. Decreasing the amount of opioids in PACU will help in deceasing the incidence of respiratory depression, constipation, opioid induced hyperalgesia, and urinary retention. Finding an adjuvant drug that is capable of decreasing consumption of opioids with minimal side effects is always an area of interest. In this study, the role of duloxetine in acute postoperative pain in patients undergoing surgery for LCS was explored with promising results. Duloxetine reduces postoperative pain after surgery as compared to a placebo, with no increase in adverse effects. 


\section{CONFLICT OF INTEREST}

No potential conflict of interest relevant to this article was reported.

\section{FUNDING}

No funding to declare.

\section{ORCID}

Nishith Govil, https://orcid.org/0000-0003-3749-6217

Kumar Parag, https://orcid.org/0000-0002-1853-003X

Pankaj Arora, https://orcid.org/0000-0002-6152-6925

Hariom Khandelwal, https://orcid.org/0000-0002-5938-4139

Ashutosh Singh, https://orcid.org/0000-0002-2214-3200

Ruchi, https://orcid.org/0000-0001-6640-6634

\section{REFERENCES}

1. Bajwa SJ, Haldar R. Pain management following spinal surgeries: an appraisal of the available options. J Craniovertebr Junction Spine 2015; 6: 105-10.

2. Shin SW, Lee JS, Abdi S, Lee SJ, Kim KH. Antipsychotics for patients with pain. Korean J Pain 2019; 32: 3-11.

3. Vilar S, Castillo JM, Munuera Martínez PV, Reina M, Pabón M. Therapeutic alternatives in painful diabetic neuropathy: a meta-analysis of randomized controlled trials. Korean J Pain 2018; 31: 253-60.

4. Turan A, Karamanlioğlu B, Memiş D, Hamamcioglu MK, Tükenmez B, Pamukçu Z, et al. Analgesic effects of gabapentin after spinal surgery. Anesthesiology 2004; 100: 935-8.

5. Onuţu AH. Duloxetine, an antidepressant with analgesic properties - a preliminary analysis. Rom J Anaesth Intensive Care 2015; 22: 123-8.

6. Sun YH, Li HS, Zhu C, Hu W, Yang J, Zhao GL, et al. The analgesia effect of duloxetine on post-operative pain via intrathecal or intraperitoneal administration. Neurosci Lett 2014; 568: 6-11.

7. Schukro RP, Oehmke MJ, Geroldinger A, Heinze G, Kress HG, Pramhas S. Efficacy of duloxetine in chronic low back pain with a neuropathic component: a randomized, double-blind, placebo-controlled crossover trial. Anesthesiology 2016; 124: 150-8.
8. Castro-Alves LJ, de Medeiros ACPO, Neves SP, de Albuquerque CLC, Modolo NS, De Azevedo VL, et al. Perioperative duloxetine to improve postoperative recovery after abdominal hysterectomy. Surv Anesthesiol 2016; 60: 171-2.

9. Gilron I. Antidepressant drugs for postsurgical pain: current status and future directions. Drugs 2016; 76: 159-67.

10. Ho KY, Tay W, Yeo MC, Liu H, Yeo SJ, Chia SL, et al. Duloxetine reduces morphine requirements after knee replacement surgery. Br J Anaesth 2010; 105: 371-6.

11. YaDeau JT, Brummett CM, Mayman DJ, Lin Y, Goytizolo EA, Padgett DE, et al. Duloxetine and subacute pain after knee arthroplasty when added to a multimodal analgesic regimen: a randomized, placebo-controlled, triple-blinded trial. Anesthesiology 2016; 125: 561-72.

12. Jacobs MB, Cohen SP. Duloxetine for subacute pain management after total knee arthroplasty: should we write it off or reevaluate? Anesthesiology 2016; 125: 454-6.

13. Smith EM, Pang H, Cirrincione C, Fleishman S, Paskett ED, Ahles T, et al.; Alliance for Clinical Trials in Oncology. Effect of duloxetine on pain, function, and quality of life among patients with chemotherapy-induced painful peripheral neuropathy: a randomized clinical trial. JAMA 2013; 309: 1359-67.

14. Castro-Alves LJ, Oliveira de Medeiros AC, Neves SP, Carneiro de Albuquerque CL, Modolo NS, De Azevedo VL, et al. Perioperative duloxetine to improve postoperative recovery after abdominal hysterectomy: a prospective, randomized, double-blinded, placebo-controlled study. Anesth Analg 2016; 122: 98-104.

15. Minami K, Tamano R, Kasai E, Oyama H, Hasegawa M, Shinohara S, et al. Effects of duloxetine on pain and walking distance in neuropathic pain models via modulation of the spinal monoamine system. Eur J Pain 2018; 22: 355-69.

16. Altiparmak B, Güzel Ç, Gümüş Demirbilek S. Comparison of preoperative administration of pregabalin and duloxetine on cognitive functions and pain management after spinal surgery: a randomized, double-blind, placebo-controlled study. Clin J Pain 2018; 34: 1114-20.

17. Kiso T, Moriyama A, Furutani M, Matsuda R, Funatsu Y. Effects of pregabalin and duloxetine on neurotransmitters in the dorsal horn of the spinal cord in a rat model of fibromyalgia. Eur J Pharmacol 2018; 827: 117-24.

18. Shen F, Tsuruda PR, Smith JA, Obedencio GP, Martin WJ. Relative contributions of norepinephrine and serotonin transporters to antinociceptive synergy between monoamine reuptake inhibitors and morphine in the rat formalin model. PLoS One 2013; 8: e74891. 